ARTICLE

Received 15 May 2015 | Accepted 5 Feb 2016 | Published 10 Mar $2016 \quad$ DOl: 10.1038/ncomms10972 OPEN

\title{
The unconventional myosin CRINKLED and its mammalian orthologue MYO7A regulate caspases in their signalling roles
}

Mariam H. Orme ${ }^{1}$, Gianmaria Liccardi ${ }^{1}$, Nina Moderau², Rebecca Feltham¹, Sidonie Wicky-John ${ }^{1}$, Tencho Tenev ${ }^{1}$, Lior Aram³, Rebecca Wilson', Katiuscia Bianchi ${ }^{1}$, Otto Morris', Celia Monteiro Domingues', David Robertson?', Meghana Tare ${ }^{4}$, Alexander Wepf ${ }^{5}$, David Williams ${ }^{6}$, Andreas Bergmann ${ }^{4}$, Matthias Gstaiger ${ }^{5}$, Eli Arama ${ }^{3}$, Paulo S. Ribeiro ${ }^{2} \&$ Pascal Meier ${ }^{1}$

Caspases provide vital links in non-apoptotic regulatory networks controlling inflammation, compensatory proliferation, morphology and cell migration. How caspases are activated under non-apoptotic conditions and process a selective set of substrates without killing the cell remain enigmatic. Here we find that the Drosophila unconventional myosin CRINKLED (CK) selectively interacts with the initiator caspase DRONC and regulates some of its non-apoptotic functions. Loss of CK in the arista, border cells or proneural clusters of the wing imaginal discs affects DRONC-dependent patterning. Our data indicate that CK acts as substrate adaptor, recruiting SHAGGY46/GSK3- $\beta$ to DRONC, thereby facilitating caspase-mediated cleavage and localized modulation of kinase activity. Similarly, the mammalian CK counterpart, MYO7A, binds to and impinges on CASPASE-8, revealing a new regulatory axis affecting receptor interacting protein kinase-1 (RIPK1) >CASPASE-8 signalling. Together, our results expose a conserved role for unconventional myosins in transducing caspase-dependent regulation of kinases, allowing them to take part in specific signalling events.

\footnotetext{
${ }^{1}$ Chester Beatty Laboratories, The Breast Cancer Now Toby Robins Research Centre, Institute of Cancer Research, Mary-Jean Mitchell Green Building, 237 Fulham Road, London SW3 6JB, UK. ${ }^{2}$ John Vane Science Centre, Centre for Tumour Biology, Barts Cancer Institute, Queen Mary University of London, Charterhouse Square, London EC1M 6BQ, UK. ${ }^{3}$ Department of Molecular Genetics, Weizmann Institute of Science, 1307 Meyer Building, Rehovot 76100, Israel. ${ }^{4}$ University of Massachusetts Medical School, 364 Plantation Street, RB Suite 419, Worcester Massachusetts 01605, USA. ${ }^{5}$ ETH, Institute for Molecular Systems Biology, Wolfgang Pauli-Strasse 16, CH-8093 Zurich, Switzerland. ${ }^{6}$ UCLA David Geffen School of Medicine, 200 Stein Plaza, Los Angeles, California 90095-7008, USA. Correspondence and requests for materials should be addressed to P.M. (email: pmeier@icr.ac.uk).
} 
$\mathrm{D}$ eregulation of caspases forms the basis of many human disease pathogeneses including neurodegeneration and cancer ${ }^{1}$. Although caspases have been extensively studied as initiators, executioners or regulators of cell death mediated by apoptosis, pyroptosis, necroptosis or autophagy ${ }^{2}$, it is clear that caspases actively regulate animal development and the defense of homeostasis through both cell death-dependent and -independent functions $s^{3-5}$. Caspase activation requires the recruitment of initiator caspases into macromolecular protein complexes that mediate the activation of initiator caspases through proximity-induced dimerization. Activation of initiator caspases depends on the engagement of platforms such as the death-inducing signalling complex, complex-II or ripoptosome for CASPASE-8 (CASP8) or CASP10, the apoptosome for CASP9 and the inflammasome for CASP-1 or -11 (ref. 6). These platforms integrate cellular signals and recruit initiator caspases via their death-fold domain, which results in the dimerization of the initiator caspases and formation of an active enzyme ${ }^{6}$. An important outstanding question is how caspases can be activated to mediate non-apoptotic events without killing the cell. Hypotheses that have been suggested include temporal restriction of activity and amplitude modulation (see ref. 3); however, it is not clear how general these modes of regulation are.

By studying how caspases take part in non-apoptotic signalling, we unexpectedly discovered an evolutionary conserved principle of caspase-mediated control of cellular processes. We find that in both Drosophila and mammals, an unconventional myosin is essential for caspase-mediated regulation of kinases. Our data demonstrate that the Drosophila myosin family member CRINKLED (CK) and its mammalian counterpart Myosin VIIA (MYO7A) act as substrate adaptor for kinases, thereby facilitating caspase-mediated cleavage and localized modulation of kinase activity. In mammals, this results in inactivation of RIPK1 and suppression of CASP8. In the absence of MYO7A, CASP8mediated cleavage and inactivation of RIPK1 is less effective. This has important implications, because mutations in MYO7A cause Usher syndrome $1 \mathrm{~B}$-an autosomal recessive disorder characterized by bilateral sensorineural hearing loss and blindness due to retinitis pigmentosa. Despite intense investigation, the mechanisms by which loss of MYO7A results in deafness and blindness are poorly understood. Our finding that MYO7A interacts with the initiator CASP8 and dampens its activation may help to explain why patients with mutations in MYO7A suffer progressive loss of sensory neurons. Given that RIPK1 and CASP8 take part in the defense of homeostasis downstream of many cytokine receptors, it is plausible that inflammatory signals contribute to the onset and progression of retinitis pigmentosa in patients with MYO7A mutations due to aberrant activation of RIPK1-dependent cell death.

\section{Results}

CK modulates DRONC-dependent phenotypes. To elucidate how caspases are regulated in their apoptotic and non-apoptotic roles, we set out to identify new binding partners of the Drosophila initiator caspase DRONC. To this end, an $\mathrm{HA}_{3 x}$-tagged form of DRONC was stably expressed in Drosophila Schneider cells (S2). DRONC protein complexes were isolated via large-scale affinity purification from Drosophila S2 cells using $\alpha$-HA resin followed by mass spectrometric analysis. As controls, we used cells stably expressing $\mathrm{HA}_{3 x}$-tagged forms of green fluorescent protein (GFP), DARK, DIAP1, DIAP2, DREDD, dTAK1, dTAB2, RELISH, KENNY and IRD5. Using DRONC as an affinity reagent, we identified a total of 89 proteins with a minimum score of two unique peptides (Supplementary Table 1). CK, the Drosophila orthologue of mammalian non-muscle MYO7A selectively co-purified with DRONC (Fig. 1a-c). Although we identified five unique CK-derived peptides in DRONC immunoprecipitates, no such peptides were identified in control immunoprecipitates, highlighting the selectivity of the CK-DRONC interaction (Supplementary Table 1). Reciprocal co-immunoprecipitation assays demonstrated that CK specifically associated with DRONC, while it did not interact with GFP, DIAP1 or drICE (Fig. 1b,c). To identify the regions of DRONC and CK that are required for their interaction, we tested several DRONC and CK fragments for their ability to bind to CK or DRONC, respectively. We found that CK preferentially bound to the CARD-containing pro-domain of DRONC (Fig. 1d). In addition, CK also weakly interacted with the p20-p10 region of DRONC, suggesting that the interaction between CK and DRONC is mediated by multiple contact points. The CARD domain of DRONC interacted with the amino-terminal portion of $\mathrm{CK}\left(\mathrm{CK}^{1-997}\right)$ encompassing the myosin head and IQ motifs (Fig. 1e), whereas the caspase domain of DRONC (p20-p10) region associated with the central region of CK $\left(\mathrm{CK}^{998-1,668}\right)$ (Fig. 1f).

CK represents an unconventional, non-muscle myosin that is highly conserved from flies to man, sharing $73 \%$ amino acid sequence similarity to its human counterpart MYO7A (Fig. 1a). MYO7A is expressed in numerous epithelial cell types, suggesting a role in multiple cellular processes. Mutations of MYO7A in humans cause Usher syndrome 1B (Fig. 1a), an autosomal recessive disorder characterized by bilateral sensorineural hearing loss and blindness due to retinitis pigmentosa ${ }^{7}$. Despite intense investigation, the mechanisms by which loss of MYO7A results in deafness and blindness are poorly understood ${ }^{8}$. In Drosophila, a loss-of-function mutation in CK $\left(c k^{13}\right)$ causes early embryonic lethality ${ }^{9}$, indicating that $\mathrm{CK}$ is indispensable for normal development. To investigate the functional significance of the interaction between CK and DRONC, we examined the requirement of CK in head involution defective (HID)mediated eye ablation (Fig. 2a). Expression of the inhibitor of apoptosis (IAP) antagonist HID in the eye activates DRONCdependent cell death, resulting in a small eye phenotype that is sensitive to the dosage of regulatory genes that control components of the apoptotic machinery ${ }^{10-17}$. Using the previously described GheF (GMR-hid ey-FLP) method $^{18}$, we found that eye-specific loss of CK $\left(c k^{13}\right.$ homozygous mutant clones in the eye) significantly protected from the GMR-hid induced eye-ablation phenotype (Fig. 2a). Importantly, loss of $c k$ function did not affect expression of HID protein, ruling out the possibility that $c k^{13}$ suppresses the HID eye phenotype by altering HID protein levels (Supplementary Fig. 1a-d). Similar to genetic deletion of $c k$, RNA interference (RNAi)-mediated knockdown of $c k$ also suppressed HID- and REAPER (RPR) killing in the eye (Supplementary Fig. 1e-h). Together, our data indicate that CK binds to DRONC and can contribute to DRONC-dependent phenotypes, such as efficient induction of cell death in response to high-level expression of the IAP antagonists HID and RPR.

To study the physiological role of CK in regulating DRONC, we investigated various paradigms of DRONC-mediated apoptotic and non-apoptotic signalling events. Rare adult 'escapers' of $c k^{13}$ mutant flies that occasionally emerge exhibit aristae, which are more highly branched than normal ${ }^{9}$ (Fig. $2 \mathrm{~b}$ and Supplementary Fig. 1i). Interestingly, branching of the Drosophila arista is regulated by DRONC ${ }^{19,20}$, an initiator caspase that is controlled by DIAP1 (ref. 11). Accordingly, surviving flies carrying the semi-lethal nonsense mutations Dronc ${ }^{24}$ or $\operatorname{Dronc}^{I 29}$ display extra aristal branches compared with wild-type controls $^{19}$. Likewise, suppression of DRONC activity via RNAimediated inactivation of Drosophila Ikk, DmIKKe (also known as $I k 2$ ), which results in an increase in DIAP1 levels and lower DRONC activity ${ }^{20}$, causes excessive branching of the arista 
a

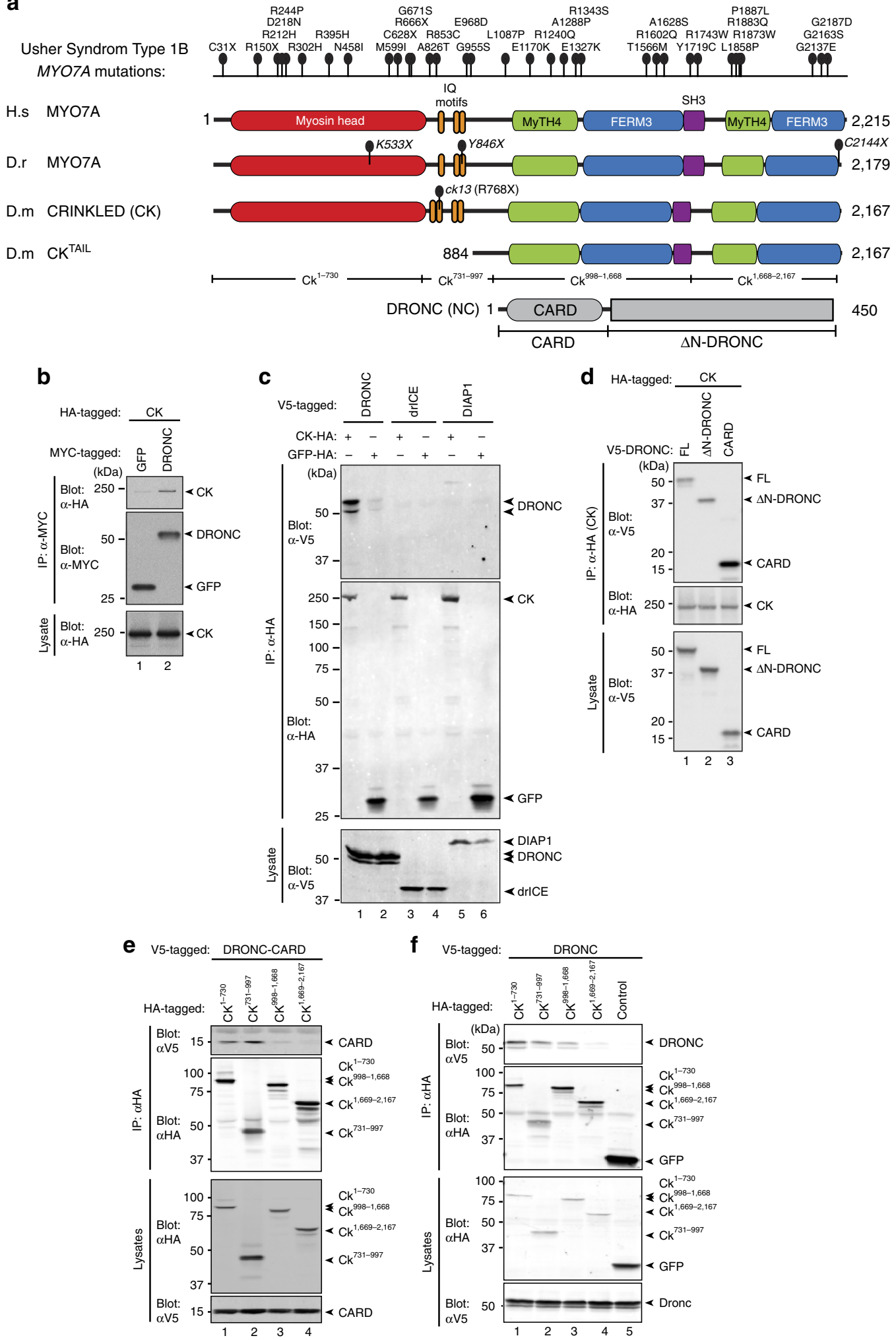

Figure 1 | CK selectively interacts with DRONC. (a) Schematic representation of MYO7A from human (Homo sapiens), zebrafish (Danio rerio) and Drosophila (D. melanogaster). Top: more than 120 mutations in the MYO7A gene have been identified in patients with Usher syndrome type 1B (http://mutdb.org/products/search). Indicated are 34 selected mutations in human MYO7A and the respective mariner mutations of zebrafish MYO7A. The loss-of-function mutation in CK $\left(c k^{13}\right)$ is indicated. (b) MYC-GFP or MYC-DRONC was co-expressed with HA-CK in Drosophila S2 cells. $\alpha$-MYC-immunoprecipitation was performed and CK interaction was assessed via immunoblotting. (c) V5-DRONC, V5-drICE or V5-DIAP1 was co-expressed with HA-CK or HA-GFP in S2 cells. $\alpha$-HA immunoprecipitation was performed and binding to CK or GFP was determined by immunoblotting. (d) V5-tagged full-length DRONC, $\triangle N$ DRONC (see scheme in a) or DRONC CARD (CARD) were co-expressed with HA-CK. $\alpha$-HA immunoprecipitation was performed and binding to CK was determined by immunoblotting. (e) V5-CARD of DRONC was co-expressed with the indicated HA-tagged fragments of CK. $\alpha-\mathrm{HA}$ immunoprecipitation was performed and binding to CK was determined by immunoblotting. (f) V5-tagged full-length DRONC was co-expressed with the indicated HA-tagged fragments of CK. $\alpha-\mathrm{HA}$ immunoprecipitation was performed and binding to CK was determined by immunoblotting. 
a

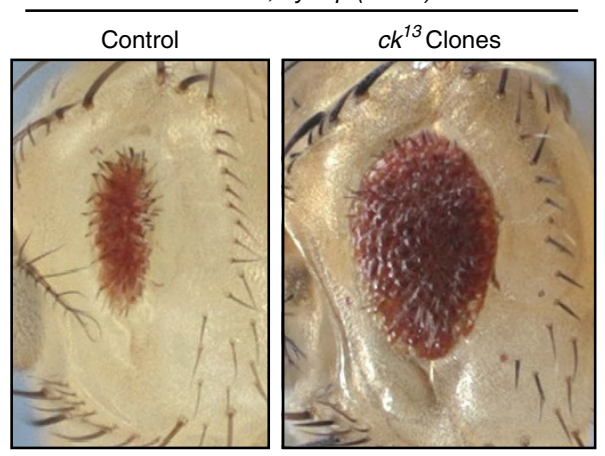

b

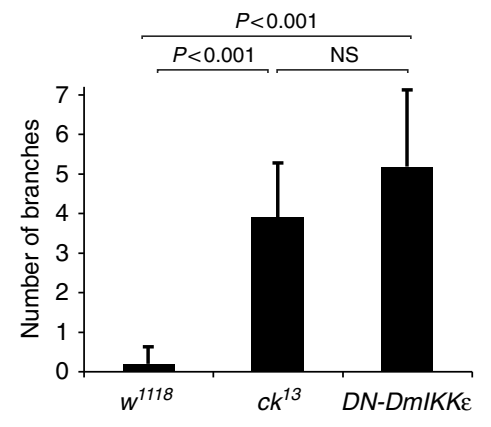

Figure 2 | Loss of CK modulates DRONC-dependent phenotypes. (a) A loss-of-function mutation in CK, ck ${ }^{13}$, suppresses the GMR-hid-induced eye phenotype. The left panel depicts the unsuppressed GMR-hid,ey-Flp (GheF) eye ablation phenotype (GMR-hid, ey-Flp/+ or Y; FRT40A/P[ubi-GFP], FRT4OA). Suppression of the GheF phenotype in ey-Flp/FRT-induced clones of $c k^{13}$ (GMR-hid, ey-Flp/ + or Y; ck $^{13}$, FRT4OA/P[ubi-GFP], FRT40A). This $c k$ allele gives rise to strong suppression of GMR-hid and is a loss-of-function mutant. (b) Mutation in ck such as expression of DN DmIKKE causes ectopic branching of the arista. Indicated is the number of branch points per lateral of the arista. First bar: $w^{1718}(n=10)$. Second bar: $c k^{13}(n=10)$. Third bar: Ap-Gal4/UAS$D m I K K \varepsilon{ }^{G 250 D}(n=11)$. Error bars represent s.d. $P$-values are $<0.001$ with respect to the control. NS indicates not significant. $P$-values were calculated using an unpaired, two-tailed $t$-test.

(Fig. 2b) (refs 9,21). Ectopic branching is also observed in hid mutants 22 . Although failure to activate DRONC results in ectopic branching, a branchless or thread-like phenotype of the arista is observed in flies that carry the DIAP1 loss-of-function mutant thread $^{122}$. This indicates that controlled activation of DRONC is required for proper arista morphogenesis. The notion that $\mathrm{CK}$ interacts with DRONC, and $c k^{13}$ and Dronc mutants display extra branches of the arista strongly suggests that $\mathrm{CK}$ regulates DRONC in the arista.

Next, we investigated the role of CK in regulating developmentally controlled or stress-induced apoptosis. A large amount of naturally occurring cell death is observed anterior to the morphogenetic furrow in the third-instar larval eye disc ${ }^{23}$ and this cell death was not suppressed in $c k^{13}$ mutant clones (Supplementary Fig. 2a,b). We also examined pupal eye development, during which apoptosis is required to remove excess interommatidial cells. We found that the number of interommatidial cells was not affected in $c k^{13}$-null clones (Supplementary Fig. 2c,d) (two-tailed $t$-test: $P=0.38$ ). Loss of $c k$ also did not affect $\mathrm{X}$-irradiation-induced DRONC activation in the wing discs of third-instar larvae (Supplementary Fig. 2e). Together, these data indicate that CK does not regulate DRONC in its apoptotic role in the fly. It is possible that although strong expression of HID and RPR promote cell death through CKassisted activation of DRONC, CK-mediated DRONC activation might not be a rate-limiting factor during naturally occurring cell death scenarios where apoptosis is executed through the concerted action of mitochondrial-dependent and -independent mechanisms ${ }^{13,24-26}$.

CK is required to suppress extra macrochaete in the scutellum. Caspase activity without cell death controls various physiological processes, including cell differentiation and cell migration ${ }^{3-5}$; however, the regulatory mechanisms of caspase activity in this context are largely unknown. With this in mind, we examined the effects of CK loss on caspase-mediated signalling events, independent of cell death. The development of sensory organ precursor (SOP) cells and the correct number of macrochaete (large bristles) on the scutellum of the notum are determined through a non-apoptotic signalling function of $\mathrm{DRONC}^{21,27}$ (Fig. 3a). Non-apoptotic DRONC activation is achieved through DmIKKE-mediated depletion of DIAP1 protein levels in cells of the proneural cluster that gives rise to SOPs. Partial depletion of DIAP1 causes caspase activation and caspase-mediated cleavage of the inactive GSK3 $\beta$ precursor SHAGGY (SGG) 46 protein. This cleavage converts it to the active kinase SGG10, which contributes to SOP cell specification ${ }^{21,27}$ (Fig. 3a), allowing the formation of precisely four macrochaete on the scutellum. However, ectopic macrochaete develop if caspase activation or activity is suppressed ${ }^{21,27}$. Thus, we examined whether loss of $c k$ phenocopies depletion of Dronc, $s g g$ and DmIKKe in specifying numbers of macrochaete in the scutellum. Knockdown of $c k$ in the scutellum, using the scabrous-GAL4 (Sca-GAL4) driver, resulted in the appearance of extra macrochaete in $32 \%$ of flies $(n=110$; Fig. $3 \mathrm{~b}, \mathrm{~d}, \mathrm{e})$. This was reminiscent of the effects of Dronc RNAi $(26 \%, n=142)$, sgg RNAi $(26 \%, n=137)$ and DmIKKE RNAi (59\%, $n=104$ ) (Fig. 3b,c,e). Interestingly, depletion of $c k$ phenocopies expression of a non-cleavable form of SGG46 $\left(\operatorname{sgg} 46^{D 235 G / D 300 G ~ 27}\right.$, thereafter referred to as $\left.\operatorname{sgg} 46^{D>G}\right)$, which causes the appearance of ectopic macrochaete in $\sim 19.3 \%$ of animals $^{27}$. This suggests that SGG46 fails to be cleaved in the absence of $c k$. Although knockdown of $c k$ resulted in extra macrochaete, RNAi against the closely related gene myo $28 B$ (the orthologue of mammalian $M y o 7 B$ ) did not lead to the formation of extra machrochaete $(n=146)$ (Fig. 3e). This suggests that $\mathrm{CK}$, but not $\mathrm{MYO} 28 \mathrm{~B}$, is required for DRONC activation in proneural clusters.

Consistent with the phenotype obtained by $c k$ RNAi, expression of a dominant-negative (DN) form of CK (CK ${ }^{\mathrm{TAIL}}$ (ref. 28)) also resulted in ectopic macrochaete in $\sim 42 \%$ of flies $(n=78)$, compared with $8 \%$ in controls $(n=165)$. Expression of $\mathrm{CK}^{\mathrm{TAIL}}$ enhanced the ectopic macroachaete phenotype caused by $D m I K K \varepsilon$ RNAi (Fig. 3e). These observations suggest that CK regulates DRONC in its non-apoptotic role in proneural clusters, to determine the correct number of SOP cells.

To gain insight into the mechanism by which $\mathrm{CK}$ regulates the number of macrochaete, we investigated whether CK might function as a substrate adaptor linking DRONC to SGG46 and facilitating its conversion to SGG10. Binding assays revealed that CK specifically co-purified SGG46 from cellular extracts, whereas it did not associate with DmIKKe under the same conditions (Fig. 4a). Intriguingly, CK bound exclusively to the inactive GSK3 $\beta$ precursor SGG46 and did not associate with SGG10 (Fig. 4b). Given that CK interacts with both DRONC and the precursor form of SGG46, and that loss of $c k$ phenocopies loss of 
a

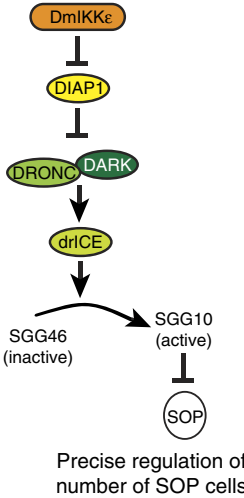

b
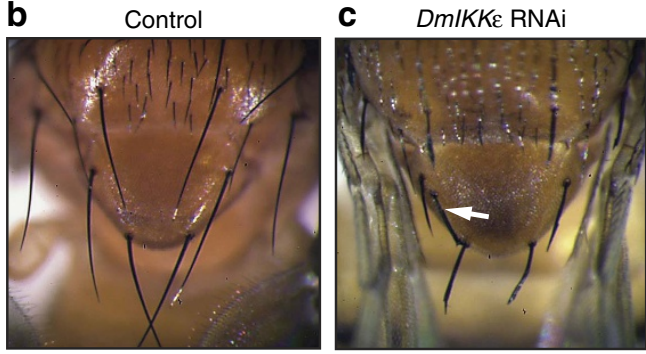

d $\quad c k$ RNAi

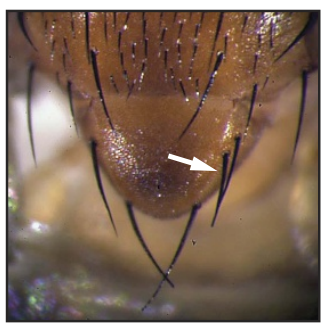

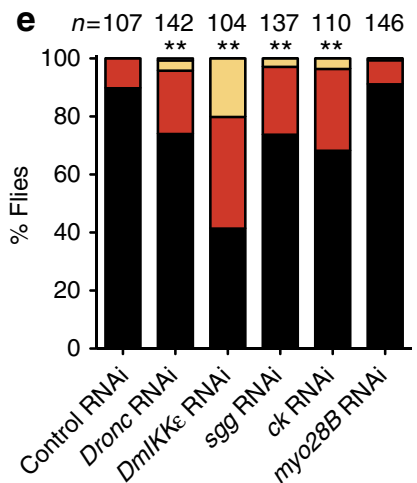

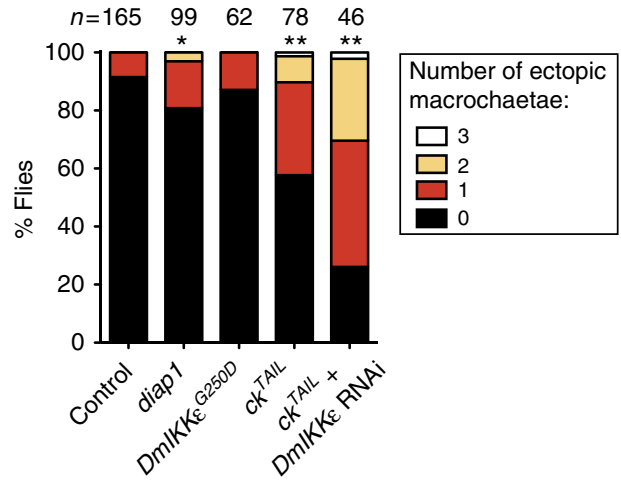

Figure 3 | CK but not its close homologue MYO28B is required for DRONC activation and the suppression of extra macrochaete in the scutellum. (a) Schematic diagram depicting the regulation of normal SOP cell number. (b-d) Light microscope images of macrochaete in the scutellum of the adult notum. White arrows indicate ectopic macrochaete. Genotypes: (b) Canton S. (c) UAS-DmIKKE-IR/sca-Gal4. (d) sca-Gal4/+; UAS-ck-IR/ + .

(e) Quantification of the number of ectopic macrochaete on the notum of adult flies of the indicated genotypes. ${ }^{*} P$-value $<0.05$ with respect to the control and ${ }^{* \star} P$-value $<0.005$ with respect to the control. P-values were calculated using Fisher's exact test. Genotypes: Control RNAi: UAS-GFP-IR/sca-Gal4. Dronc RNAi: UAS-Dronc-IR/sca-Gal4. DmIKKe RNAi: as in c. sgg RNAi: UAS-sgg-IR/sca-Gal4. ck RNAi as in d. myo28B RNAi: UAS-myo28B-IR/sca-Gal4.

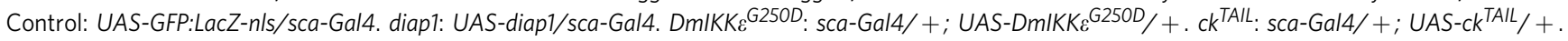
$c k^{T A I L}+$ DmIKKE RNAi: UAS-DmIKKE-IR/sca-Gal4; UAS-ckTAIL $/+$.

a

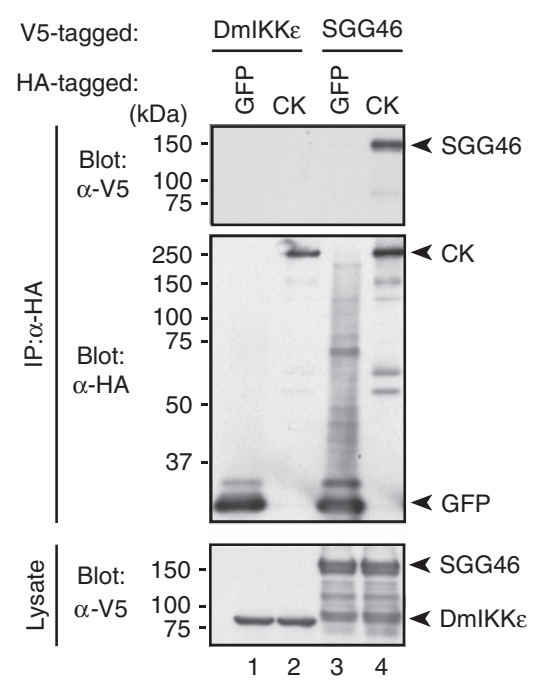

b
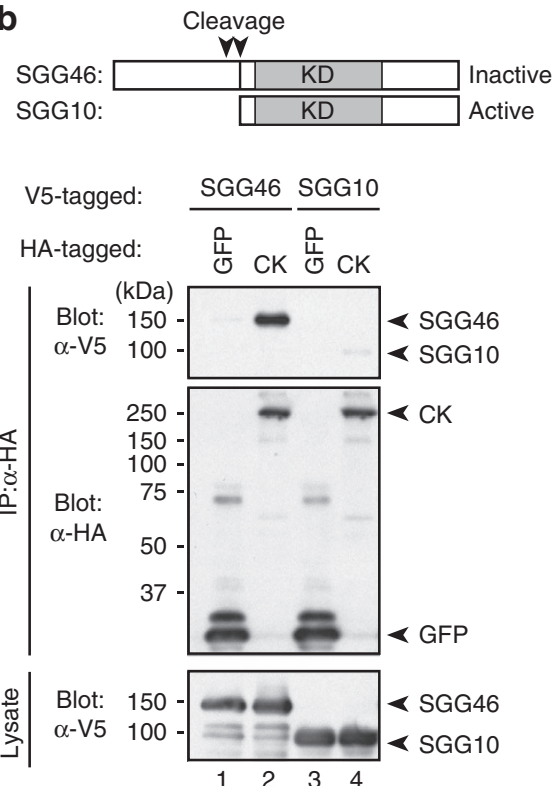

Figure 4 | CK interacts with SGG46. (a) V5-DmIKKE or V5-SGG46 was co-expressed with HA-GFP or HA-CK in Drosophila S2 cells. $\alpha$-HAimmunoprecipitation was performed and CK interaction was assessed via immunoblotting. (b) V5-SGG46 or V5-SGG10 was co-expressed with HA-GFP or HA-CK in Drosophila S2 cells. $\alpha$-HA-immunoprecipitation was performed and CK interaction was assessed via immunoblotting. Top panel: schematic diagram indicating the drICE cleavage sites on SGG. KD, kinase domain.

Dark, Dronc and sgg or expression of $\mathrm{SGG} 46^{\mathrm{D}}>\mathrm{G}$, our data suggest a mechanism whereby $\mathrm{CK}$ acts as a substrate adaptor bringing SGG46 in close proximity to the caspase, thereby facilitating cleavage and localized activation of SGG46. According to this scenario, following caspase-mediated cleavage, active SGG10 is then released to regulate SOP cell specification. To gain a better understanding why $\mathrm{CK}^{\mathrm{TAIL}}$ acts as a DN mutant, we tested the ability of $\mathrm{CK}^{\text {TAIL }}$ to bind to DRONC. As shown in 
Supplementary Fig. 3a, CK ${ }^{\text {TAIL }}$ bound to DRONC as efficiently as wild-type CK. As CK helps to recruit substrates (such as SGG46) to DRONC, it is likely to be that $\mathrm{CK}^{\mathrm{TAIL}}$ fails to associate with caspase substrates. Thus, $\mathrm{CK}^{\mathrm{TAIL}}$ may function as $\mathrm{DN}$, because it prevents the recruitment of caspase substrates to DRONC. Validation of how CK modulates DRONC signalling will ultimately require the reconstitution of stable DARK/DRONC/ drICE/CK/SGG46 protein complexes. Attempts to reconstitute this complex in vitro have been hampered by difficulties in producing recombinant $\mathrm{CK}$ due to its sheer size (2,176 amino acids).

DN forms of CK rescue the $\operatorname{Rac}^{N 17}$ migration defect. The migration of border cells in the developing egg chamber is another non-apoptotic developmental process in which DmIKKe and DRONC have been implicated ${ }^{29}$. Border cell migration provides a simple model to study how cells that originate within an epithelial monolayer subsequently become migratory. Border cells undergo 'collective cell migration' from the anterior pole of the developing egg chamber towards the oocyte, reaching their destination by stage 10 of oocyte development. The collective migration of border cells is dependent on the activity of the small GTPase RAC. Expression of a DN form of RAC $\left(\mathrm{RAC}^{\mathrm{N} 17}\right)$ almost entirely blocked migration of the border cell cluster ${ }^{29}$ (Fig. 5a,b,d,f,h,i). Importantly, this migration defect can be rescued through suppression of DRONC activity, either by mutations affecting DARK, expression of a DN form of DRONC $\left(\mathrm{DRONC}^{\mathrm{DN}}\right)$ or expression of DIAP1 (ref. 29). The role for DRONC in negatively regulating RAC-mediated cell motility represents an apoptosis-independent function of DRONC. Given that the migratory phenotype of $\mathrm{RAC}^{\mathrm{N} 17}$-expressing border cells is sensitive to DRONC activity, we assessed whether CK is required for DRONC activation in border cells. Normal border cells complete migration to $100 \%$ at stage 10 (Fig. $5 \mathrm{c}, \mathrm{h}, \mathrm{i}$ ). In contrast, slbo-GAL4-driven expression of UAS-Rac ${ }^{N 17}$ resulted in severe migration defects in which none of the border cell clusters a
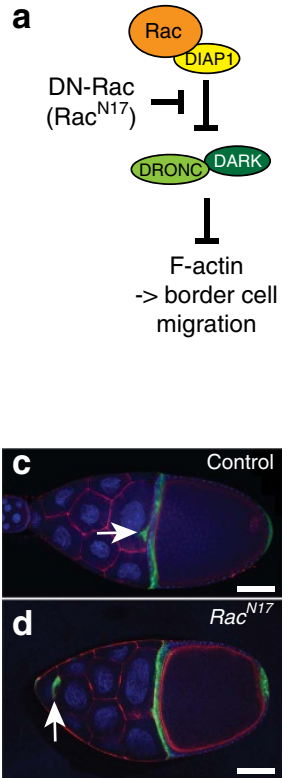

e
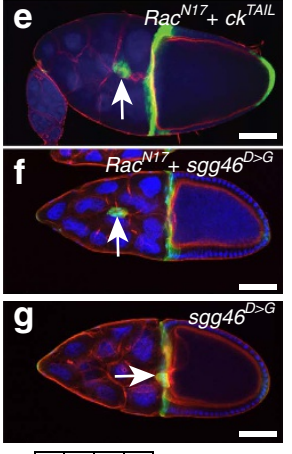

$0 \longdiv { 2 5 5 0 7 5 1 0 0 \% }$

Distance moved b

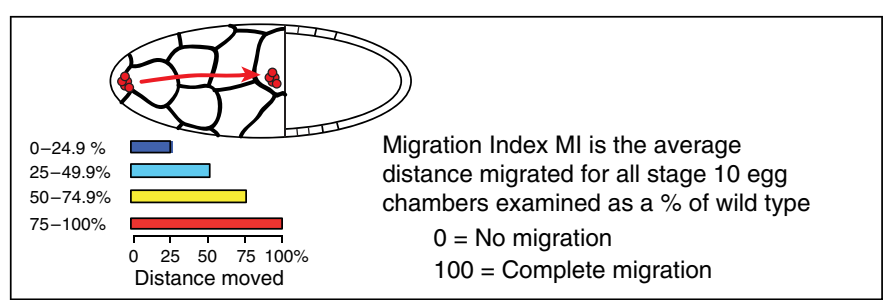

h
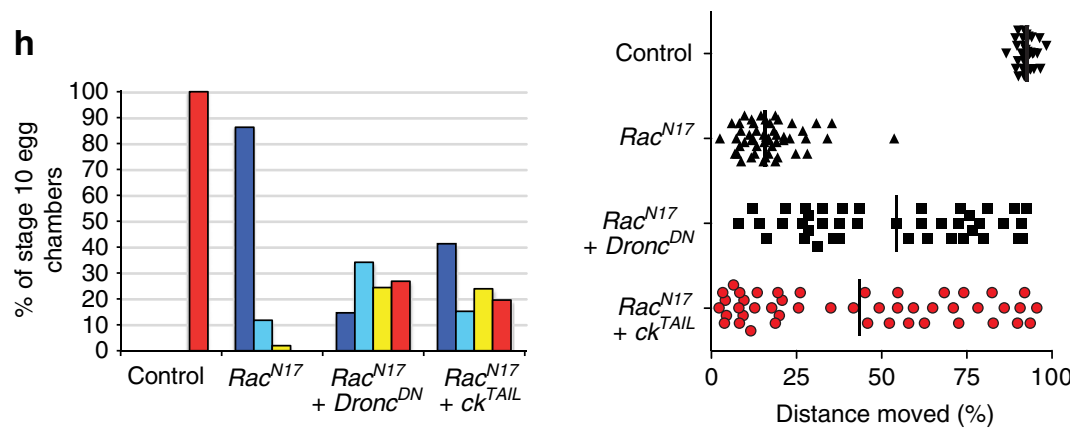

MI:

100

17

59

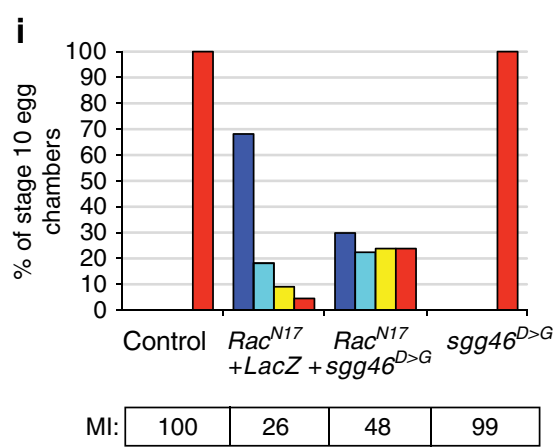

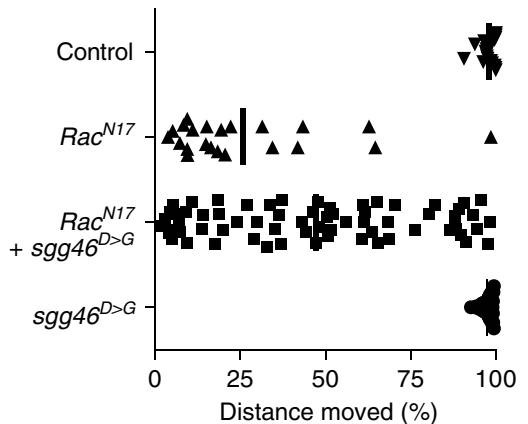

Figure 5 | Expression of DN forms of CK rescues the RAC ${ }^{\mathbf{N 1 7}}$ migration defect. (a) Schematic diagram depicting Rac-mediated regulation of border cell migration. (b) Schematic drawing of a stage-10 egg chamber. Border cells and their moving trajectory are indicated in red. (c-g) Confocal microscope images of stage-10 egg chambers of the indicated genotypes. Border cell clusters are indicated by arrows. Blue: DAPI (4,6-diamidino-2-phenylindole). Green: GFP, indicating border cells. Red: Phalloidin. Scale bars, $50 \mu \mathrm{m}$. Genotypes: (c) slbo-Gal4, UAS-GFP/ +. (d) slbo-Gal4, UAS-GFP/ + ; UAS-Rac N17/ +. (e) slbo-Gal4, UAS-GFPI +; UAS-Rac N17/UAS-ck TAll. (f) slbo-Gal4, UAS-GFP/UAS-MYCsgg46 D235G/D300G; UAS-Rac N17/ +. (g) slbo-Gal4, UAS-GFP/ UAS-MYCsgg46 ${ }^{\text {D235G/D300G }}++/+$. (h,i) Rescue of border cell migration defects. Graphs showing the distance moved by border cells in egg chambers from flies of the indicated genotypes. The data are plotted using a bar diagram (left) or scatter plot (right). Bars in the scatter plot represent the median values. Genotypes: control: as in c. Rac N17. (h): as in d. Rac N17 + LacZ. (i) slbo-Gal4, UAS-GFP/UAS-LacZ;UAS-Rac N17/ +. Rac N17 + Dronc DN: slbo-Gal4, UAS-GFPI + ; UAS-Rac N17/UAS-Dronc ${ }^{C>A}$. Rac $^{N 17}+c k^{\text {TAll }}$ : as in e. $\operatorname{Rac}^{N 17}+s g g 46^{D>G}$ : as in f. sgg46 ${ }^{D>G}$ : as in $\mathbf{g}$. 
reached their final destination (Fig. 5d,h,i). Less than $2 \%$ of $\mathrm{RAC}^{\mathrm{N} 17}$-expressing egg chambers had border cell clusters that reached halfway to their presumptive destination. Importantly, expression of $\mathrm{CK}^{\mathrm{TAIL}}$ suppressed the $\mathrm{RAC}^{\mathrm{N} 17}$ migration defect significantly, with $43.5 \%$ of egg chambers having border cells that migrated at least halfway to the oocyte (Fig. 5e,h). The ability of $\mathrm{CK}^{\mathrm{TAIL}}$ to rescue the $\mathrm{RAC}^{\mathrm{N} 17}$ was comparable to that of $\mathrm{DRONC}^{\mathrm{DN}}$, expression of which resulted in $51.2 \%$ of egg chambers having border cells that migrated at least halfway to the oocyte (Fig. 5h). Although no RAC ${ }^{\mathrm{N} 17}$ egg chambers had border cells that migrated beyond $75 \%$ of the total distance, expression of DN forms of CK and DRONC rescued the $\mathrm{Rac}^{\mathrm{N} 17}$ phenotype, allowing border cells in $19.6 \%$ and $26.8 \%$, respectively, of the egg chambers to migrate further than $75 \%$ of their distance. Given that $\mathrm{CK}^{\mathrm{TAIL}}$ phenocopies $\mathrm{DRONC} \mathrm{CN}^{\mathrm{DN}}$ in rescuing the $\mathrm{RAC}^{\mathrm{N} 17}$ migration phenotype, our data further demonstrate a functional interaction between CK and DRONC, and are consistent with the notion that $\mathrm{CK}$ contributes to DRONC-mediated non-apoptotic signalling.

Next, we addressed whether CK affects caspase signalling through a common mechanism in these multiple models of caspase function. During SOP specification, CK seems to function as substrate adaptor that helps to bridge DRONC and SGG46. To test the role of SGG46 cleavage in border cell migration, we expressed SGG46 $^{\mathrm{D}>\mathrm{G}}$ that cannot be cleaved ${ }^{27}$ (Fig. 5f,g,i). If the RAC ${ }^{\mathrm{N17}}$ migration defect was mediated by DRONC-dependent cleavage of SGG46, the prediction is that a non-cleavable form of SGG46 would phenocopy DRONC ${ }^{\mathrm{DN}}$ and $\mathrm{CK}^{\mathrm{TAIL}}$, and rescue the $\mathrm{RAC}^{\mathrm{N} 17}$ block in migration. Indeed, expression of $\mathrm{SGG} 46^{\mathrm{D}>\mathrm{G}}$ rescued the $\mathrm{RAC}^{\mathrm{N17}}$ migration phenotype (Fig. 5f,g,i). Importantly, the ability of $S G G 46^{D}>\mathrm{G}$ to rescue the RAC $^{\mathrm{N} 1}$ phenotype was comparable to the effect of DRONCDN and $\mathrm{CK}^{\mathrm{TAIL}}$ (Fig. 5 and also compare Fig. 5h with Fig. 5i). This also demonstrates that cleavage of a single substrate is sufficient to mediate the RAC ${ }^{\mathrm{N} 17}$ phenotype. Given that $\mathrm{SGG} 46^{\mathrm{D}}>\mathrm{G}$ phenocopies $\mathrm{CK}^{\mathrm{TAIL}}$ and DRONC ${ }^{\mathrm{NN}}$ in rescuing the RAC ${ }^{\mathrm{N} 17}$ migration phenotype, our data are consistent with the notion that $\mathrm{RAC}^{\mathrm{N} 17}$ drives CK-mediated activation of DRONC, which results in caspase-dependent cleavage and activation of SGG46. Activation of SGG subsequently suppresses border cell migration, most probably by influencing the dynamics of actin cytoskeleton, microtubule and adhesion turnover.

We further investigated the role of $\mathrm{CK}$ in regulating two other caspase-dependent signalling pathways, Drosophila innate immune signalling and spermatid individualization. We found that CK specifically interacted with the death-domain-containing protein immune defiency (IMD) and ameliorated IMD signalling following septic injury with the Gram-negative bacteria Ecc15 (Supplementary Fig. 3b-d). This is consistent with an earlier report implicating $\mathrm{CK}$ as a putative negative regulator of IMD signalling ${ }^{30}$. Although $C K$ regulates initiator caspases in their non-apoptotic roles in border cells, SOP and arista, CK appeared to play no rate-limiting role in DRONC-mediated individualization of spermatids (Supplementary Fig. 3e). Together, our data demonstrate that CK regulates caspases in some but not all signalling aspects. Quite possibly, SGG46 is only involved in border cells, SOP, arista and fat body cells (for IMD signalling), but not in spermatids.

MYO7A binds to CASP8 in a RIPK1-dependent manner. Human MYO7A shares $73 \%$ amino acid sequence similarity to Drosophila CK. Mutations in MYO7A cause Usher syndrome type $1 \mathrm{~B}$, a disease characterized by the combination of sensorineural hearing loss and visual impairment termed retinitis pigmentosa ${ }^{7}$. Previous studies of the mutant zebra fish mariner, which carries a mutation in myo7aa (the zebra fish homologue of MYO7A), revealed mild endoplasmic reticulum (ER) stress and sensory neuron apoptosis ${ }^{31}$. However, the mechanism through which such MYO7A alleles render cells susceptible to apoptosis remains ill defined. Given that CK directly interacts with the initiator caspase DRONC, we assessed whether MYO7A might bind and regulate caspases. To this end, we tested the ability of mammalian MYO7A to associate with CASP8, CASP9 and the effector CASP3. Intriguingly, we found that MYO7A selectively bound to CASP8, whereas it failed to associate with CASP3 or CASP9 under the same experimental conditions (Fig. 6a). The binding of CASP8 to MYO7A was mediated by the death effector domain (DED) of CASP8, as this domain in isolation specifically interacted with MYO7A (Fig. 6b). Consistently, deletion of the DED ( $\triangle \mathrm{N}$-CASP8) completely abrogated the binding of CASP8 to MYO7A, indicating that MYO7A only binds to pro-CASP8.

To investigate the localization and interaction of endogenous MYO7A and CASP8, we performed immunofluorescence confocal microscopy. Staining with MYO7A- and CASP8-specific antibodies revealed that the expression of endogenous MYO7A closely overlapped with that of CASP8 (Fig. $6 c$ and Supplementary Fig. 4a). Two different $\alpha$-CASP8 antibodies, when combined with $\alpha$-MYO7A antibodies, provided identical results (compare Fig. $6 \mathrm{c}$ with Supplementary Fig. 4a).

To visualize MYO7A/CASP8 protein interaction in their native state in intact cells, we applied in situ proximity ligation assay $(\text { PLA })^{32}$ with a combination of antibodies that generate a localized, discrete signal only when CASP8 and MYO7A are in close proximity, that is, in a complex. Using specific primary antibodies against CASP8 and MYO7A, which in turn were recognized by oligonucleotide-coupled secondary antibodies, we obtained discrete proximity labelling of CASP8 and MYO7A, confirming their close proximity (Fig. 6d). Virtually, the same result was obtained using two different primary $\alpha$-CASP8 antibodies (Fig. 6d and Supplementary Fig. 4b). To control for nonspecific binding between the various secondary antibodies, we added only one or no primary antibody to the reaction and observed no signal (Fig. 6d). Intriguingly, MYO7A/CASP8 proximity labelling was entirely dependent on the presence of RIPK1. Accordingly, depletion of Ripk1 by RNAi completely abolished CASP8/MYO7A proximity signals (Fig. 6d, compare $\mathrm{d}(\mathrm{ii})$ and $\mathrm{d}(\mathrm{iii})$ with $\mathrm{d}(\mathrm{iv}))$. This result also demonstrates that the observed proximity signal is not due to nonspecific binding of the primary antibodies. The requirement of RIPK1 for the generation of CASP8/MYO7A proximity signals strongly suggests that CASP8 and MYO7A interact with one another within RIPK1based protein complexes such as the ripoptosome.

MYO7A suppresses ripoptosome formation. RIPK1 can form protein complexes in the cytosol with CASP8, cFLIP (cellular FLICE-like inhibitory protein) and Fas-associated protein with a death domain (FADD). Depending on the signals that stimulate the assembly of RIPK1-based protein complexes, this complex is referred to as complex-II or ripoptosome ${ }^{33-37}$. Active CASP8 within complex-II/ripoptosome cleaves and inactivates RIPK1, thereby counteracting the assembly and stability of this complex ${ }^{38,33}$. The complex-II/ripoptosome is further negatively regulated by the E3 ubiquitin-ligases cIAP1, cIAP2 and XIAP, which target this complex for Ub-dependent inactivation ${ }^{33}$. When IAPs and/or CASP8 are inhibited, complex-II/ripoptosome complexes significantly accumulate and are stabilized ${ }^{40}$. Consistent with the notion that PLA can be adapted to detect complex-II/ripoptosome in situ, we found that specific primary antibodies against RIPK1 and CASP8 resulted in RIPK1/CASP8 proximity signals (Fig. 7a-i). Importantly, these proximity signals were significantly enhanced when IAPs and/or CASP8 were inhibited, such as following 

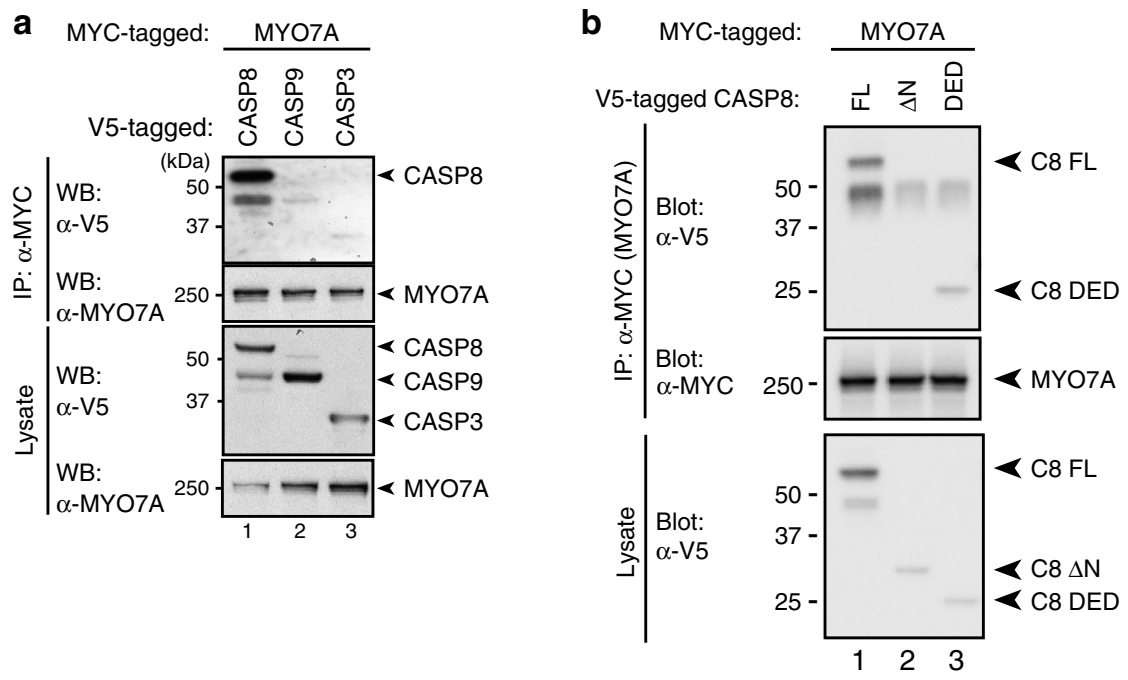

C

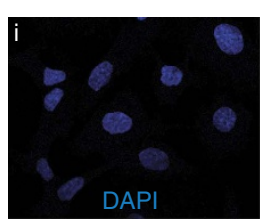

Confocal immunofluorescence microscopy
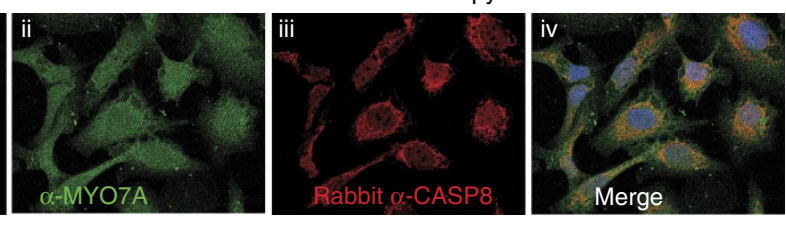

d

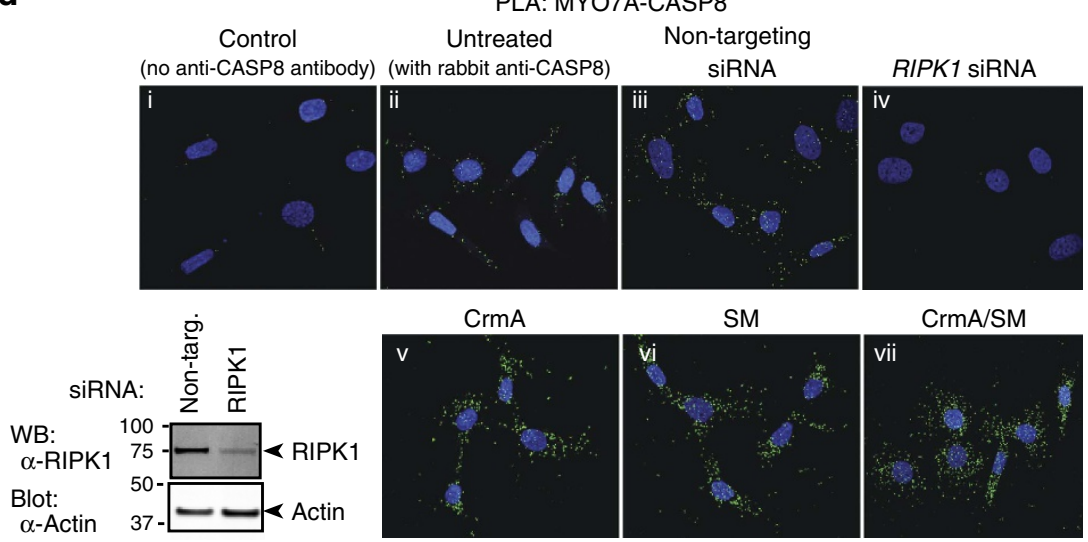

PLA: MYO7A-CASP8

Figure 6 | MY07A selectively binds to CASP8. (a) MYC-MYO7A was co-expressed with V5-CASP8, V5-CASP9 or V5-CASP3 in 293T cells. $\alpha$-MYC-immunoprecipitation was performed and MYO7A interaction was assessed via immunoblotting. (b) The indicated proteins were co-expressed in 293T cells and the interaction between MYO7A and full-length or deletion mutants of CASP8 was assessed by immunoprecipitation followed by immunoblotting. (c) Confocal microscopy images of SKOV3 cells showing co-localization of MYO7A (green) and CASP8 (red) using the indicated antibodies. Nuclei stained with DAPI (4,6-diamidino-2-phenylindole; blue). (d) Representative confocal microscopy images of proximity ligation assay (PLA) using $\alpha$-MYO7A and either no $\alpha$-CASP8 antibody (i) or rabbit $\alpha$-CASP8 antibody (i-vii) in SKOV3 cells. PLA signals are in green and nuclei are stained with DAPI (blue). PLA between MYO7A and CASP8 on siRNA knockdown of control (non-targeting oligos) (iii) or RIPK1 (iv). Immunoblot analysis of RIPK1 and ACTIN following siRNA knockdown of control or Ripk1. PLA between MYO7A and CASP8 in cells stably expressing CrmA (v), on treatment with $100 \mathrm{nM} \mathrm{SM}$ for $5 \mathrm{~h}$ (vi), or in response to SM/CrmA treatment (vii).

treatment with pharmacological inhibitors of IAPs (second mitochondria-derived activator of caspase mimetic, SM) (Fig. 7a(ii)) and stable expression of the viral CASP8 inhibitor CrmA (Fig. 7a(iii)). This is entirely consistent with co-immunoprecipitation experiments, demonstrating that the ripoptosome is stabilized following treatment with SM or CASP8 inhibitors ${ }^{33,34}$. The notion that the proximity technique reliably detects complexII/ripoptosome formation in situ is further corroborated by the observation that treatment with tumour necrosis factor (TNF)/ SM, which drives RIPK1-dependent formation of complex-II (ref. 35 ), resulted in a dramatic increase in RIPK1/CASP8 proximity signals within a few hours (Fig. 7b).
TNF is a pleiotropic cytokine inducing a variety of cellular responses ranging from inflammatory cytokine production, cell survival, cell proliferation and, paradoxically, CASP8-dependent cell death ${ }^{41,42}$. Although it is clear that many human pathogeneses are caused by deregulated TNF signalling, recent evidence suggests that some TNF-driven diseases might not only be due to aberrant TNF-mediated activation of nuclear factor- $\kappa \mathrm{B}$ and the production of cytokines ${ }^{43}$, but also might be the result of deregulated TNF-induced cell death ${ }^{44}$. In this respect, it is interesting to note that TNF contributes to the neurotoxicity observed in retinal neurodegenerative disorders ${ }^{45}$. Given that mutations in MYO7A sensitize cells to apoptosis and retinitis 
a

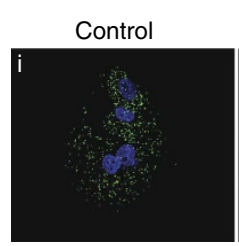

C
PLA: RIPK1-CASP8 SM

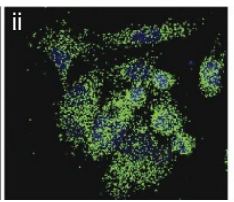

CrmA

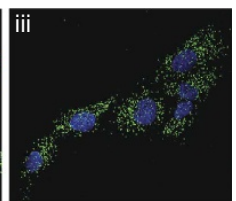

b

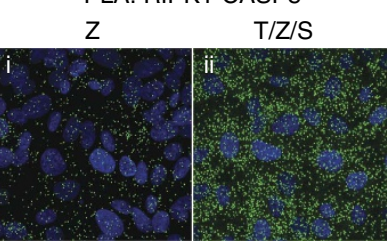

PLA: RIPK1-CASP8

PLA

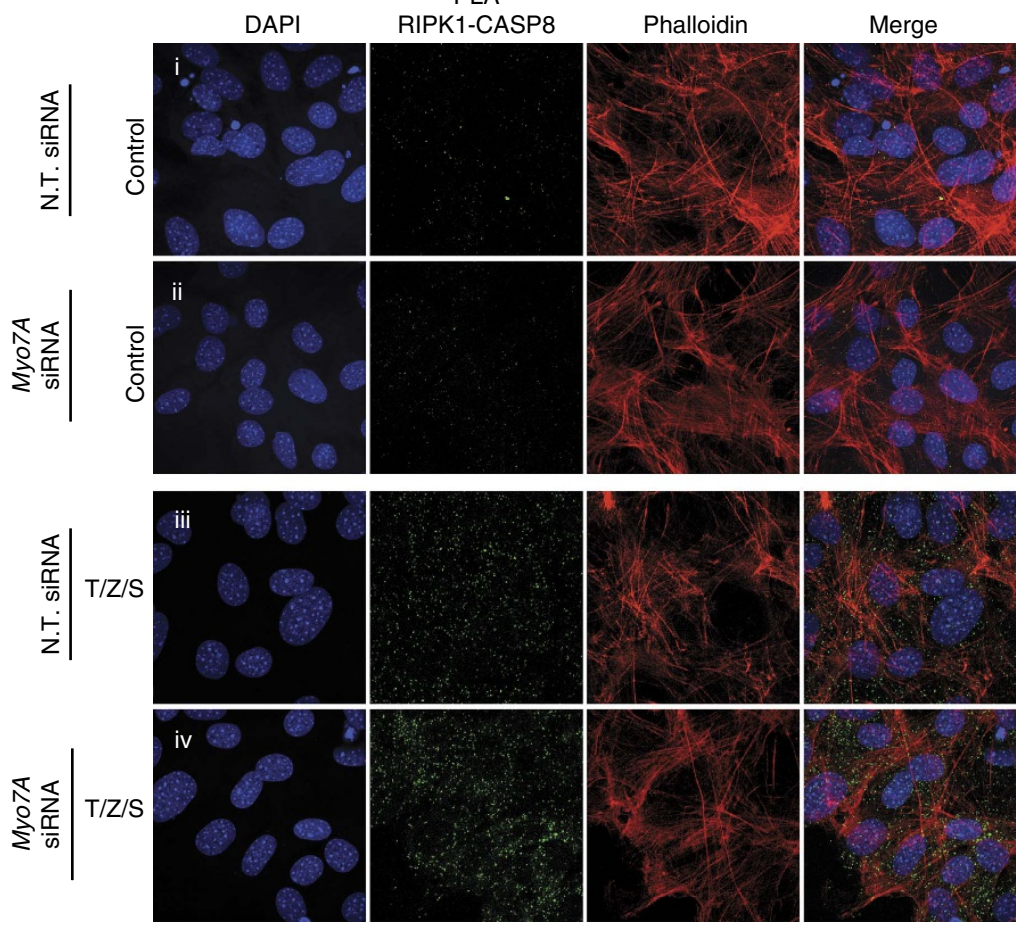

Figure 7 | MYO7A suppresses ripoptosome formation. (a) Proximity ligation assay (PLA) between RIPK1 and CASP8 in SKOV3 cells on control treatment (i), $100 \mathrm{nM}$ SM for $5 \mathrm{~h}$ or in cells stably expressing CrmA (iii). (b) PLA between RIPK1 and CASP8 in HT1080 cells on treatment with zVAD (i) or TNF/SM/ zVAD (ii). (c) PLA between RIPK1 and CASP8 in SWISS-3T3 cells on the indicated treatments. Nuclei are stained with DAPI (4,6-diamidino-2-phenylindole; blue), PLA signals are in green and Phalloidin staining is in red.

pigmentosa $^{31,46}$, and that MYO7A can interact with CASP8 in a RIPK1-dependent manner (Fig. 6d(iv)), we assessed whether MYO7A modulates TNF-dependent formation of complex-II/ ripoptosome. Intriguingly, we found that RNAi-mediated knockdown of MYO7A enhanced TNF-induced formation of RIPK1/CASP8 complexes (Fig. 7c and compare Fig. 7c(iii) with Fig. $7 c($ iv) ).

Loss of MYO7A sensitizes cells to cytokine-induced cell death in a CASP8-dependent manner. Consistent with the notion that depletion of MYO7A caused enhanced formation of RIPK1/ CASP8 complexes, we found that this was accompanied with enhanced processing and activation of CASP8, elevated levels of effector caspase activity (DEVDase) and reduced cell viability (Fig. 8a-c and Supplementary Fig. 4). Under the same conditions, knockdown of Casp8 or Ripk1 suppressed caspase activation (Fig. 8 and Supplementary Fig. 4), demonstrating that the enhanced cytotoxic effect of TNF on depletion of MYO7A is RIPK1 and CASP8 dependent. In all cell lines tested, knockdown of MYO7A enhanced TNF-induced caspase activation and cell death (Supplementary Fig. 4), extending this observation to other cell types. Therefore, these data indicate that MYO7A contributes to the proper regulation of CASP8, protecting cells from the cytotoxic effects of cytokines such as TNF. The observation that MYO7A binds to the DED of CASP8 (Fig. 6b) is particularly intriguing, because for full activation CASP8 requires sequential interaction of CASP8 molecules, via their DED domains, to form a caspase-activating chain ${ }^{47}$. As MYO7A binds to the DED of CASP8 and dampens its activation, it is possible that MYO7A suppresses the formation of CASP8 chains, thereby acting as a chain terminator. Alternatively, MYO7A might regulate the stability/turnover of complex-II/ripoptosome. A characteristic feature of complex-II/ripoptosome is that it regulates its own stability $^{38,39}$. Once RIPK1 adopts a binding-competent state, it recruits FADD, CASP8 and $\mathrm{CFLIP}_{\mathrm{L}}$. FLIP $_{\mathrm{L}}$-mediated activation of CASP8 results in cleavage of RIPK1, which results in disassembly of the complex. Given that depletion of MYO7A results in enhanced complex formation (Fig. $7 c$ (iv)), it is possible that the presence of MYO7A favours CASP8-mediated cleavage of RIPK1 and disassembly of the complex. This would be reminiscent to the situation in Drosophila where CK helps DRONC to drive the cleavage of SGG46. In the absence of MYO7A, less RIPK1 would be cleaved, which results in a build-up of complex-II/ripoptosome formation (as evidenced in Fig. 7c(iv)), which in turn would lead to enhanced caspase activation. According to this scenario, MYO7A would not actually change the activity of CASP8 but alter the stability of complex-II/ripoptosome. These two models 
a

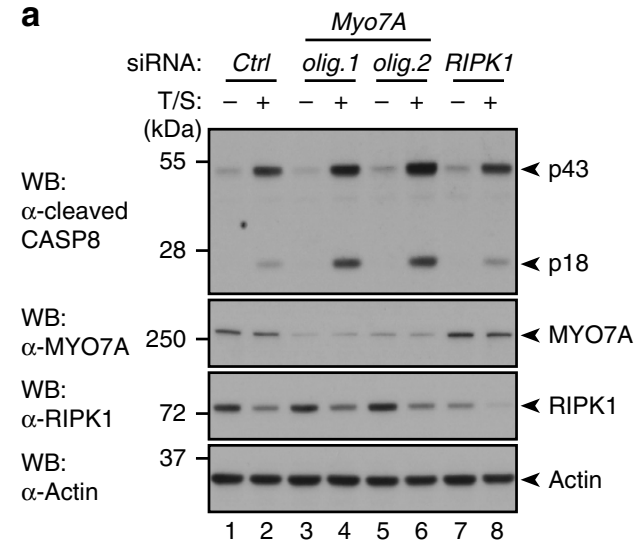

b

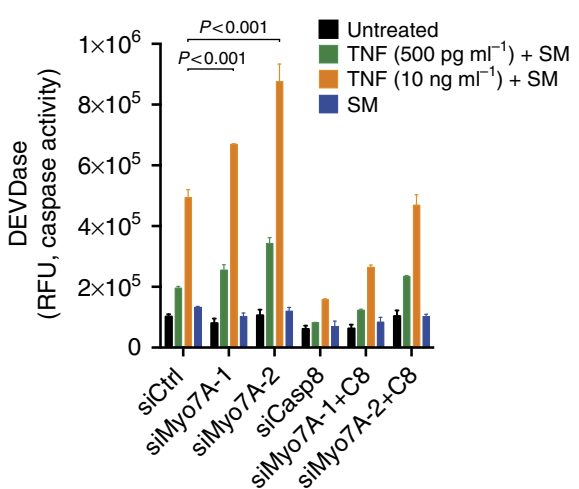

c

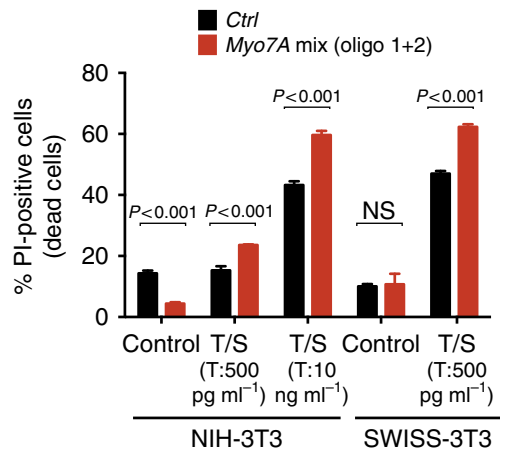

Figure 8 | Loss of MYO7A sensitizes cells to cytokine-induced cell death in a CASP8-dependent manner. (a) WB analysis of activated CASP8 (p43 and p18 cleavage products), MYO7A, RIPK1 or ACTIN following the indicated treatments in NIH-3T3 cells. Depicted are representative westernblot images of three biological repeats. (b) DEVDase assays from lysates of NIH-3T3 cells subjected to siRNA targeting Myo7A or Casp8, and exposed to the indicated treatments. (c) FACS analysis of PI-positive NIH-3T3 or SWISS-3T3 cells subject to siRNA knockdown of the indicated genes followed by the indicated treatments. Error bars represent s.d. P-values are indicated. Experiments were conducted in triplicates.
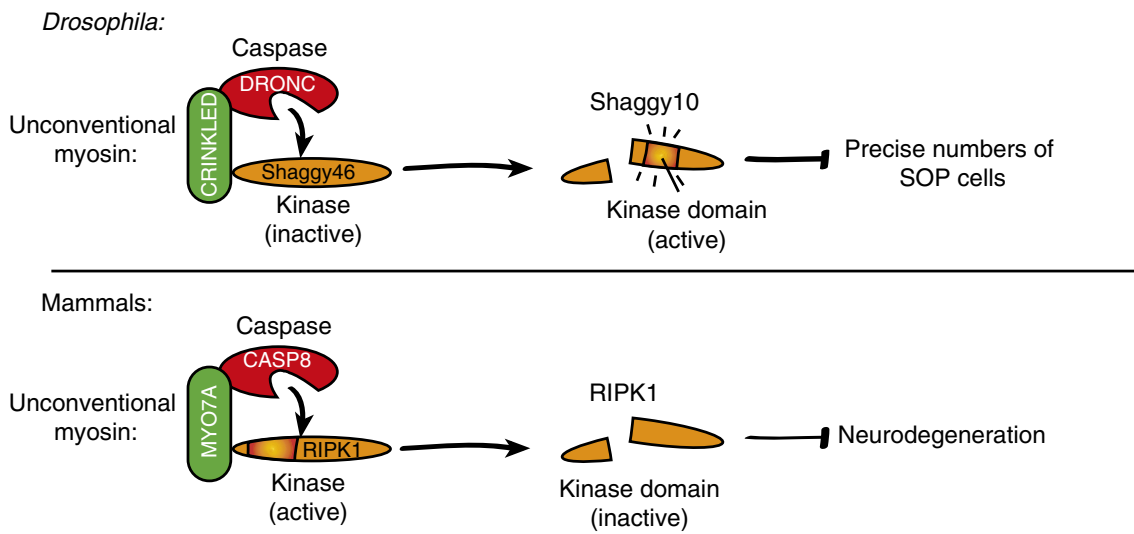

Figure 9 | Schematic model depicting the evolutionary conserved and unifying principle of caspase-mediated regulation of kinases. In Drosophila, binding of CK to DRONC favours DRONC-mediated cleavage and maturation of SGG46 into its active form SGG10. In mammals, MYO7A might regulate CASP8-mediated cleavage of RIPK1, which results in disassembly of the ripoptosome complex. In the absence of MYO7A, less RIPK1 would be cleaved, which results in a build-up of complex-II/ripoptosome formation, which in turn would lead to enhanced caspase activation.

are not mutually exclusive, as both mechanisms could operate simultaneously. Ultimately, quantitative and structural mass spectrometric approaches will be necessary to determine the stoichiometry of RIPK1, FADD, CASP8 and $\mathrm{FLIP}_{\mathrm{L}}$ in the presence and absence of MYO7A.

\section{Discussion}

Usher syndrome, the leading cause of hereditary combined hearing and vision loss, is characterized by sensorineural deafness and progressive retinal degeneration ${ }^{7}$. Mutations in several different genes produce Usher syndrome; however, the proximal cause of sensory cell death remains unknown. Our finding that MYO7A interacts with the initiator CASP8 and dampens its activation may help to explain why patients with mutations in MYO7A suffer progressive loss of sensory neurons. Given that RIPK1 and CASP8 take part in the defense of homeostasis downstream of many cytokine receptors, it is possible that inflammatory signals such as TNF contribute to the onset and progression of retinitis pigmentosa due to aberrant activation of RIPK1-dependent cell death. Our findings have important implications, as they suggest that anti-inflammatory biologics, such as Enbrel (a TNF blocker), might help to delay or even prevent the onset of this debilitating disease. Although CASP8 can initiate apoptosis downstream of TNF death receptors, the normal physiological function of CASP8 is to transmit a pro-survival signal that suppresses RIPK1-dependent necroptosis. Suppression of RIPK1-dependent necroptosis by CASP8 requires its catalytic activity. One of the key substrates processed by CASP8 is RIPK1. This destabilizes RIPK1-based death complexes ${ }^{38,39}$. The observation that RIPK1-based platforms accumulate on depletion of MYO7A might indicate that CASP8-mediated cleavage and inactivation of RIPK1 is less effective in the absence of MYO7A. This is highly reminiscent of the situation in Drosophila, where CK assists DRONC in cleaving the kinase SGG46. Thus, in both Drosophila and mammals, CK and MYO7A appear to take part in caspase-mediated regulation of kinases, revealing a unifying principle of caspase-mediated regulation of cellular processes (Fig. 9).

\section{Methods}

Reagents. Constructs were generated by PCR and cloned into pAc, pMT, pcDNA3 (Invitrogen) or pEF6 and verified by sequencing. Specific point mutations were generated using site-directed mutagenesis with Pfu Turbo polymerase (Stratagene). The Ub-sgg10 fusion construct was generated by PCR using Easy-A polymerase and cloned into pMT-V5/His. The following antibodies were used: 
$\alpha$-MYC (Sigma, M5546, 1:2,000), $\alpha$-HA (Roche, 11867423001, 1:2,000), $\alpha$-V5 (Serotec, MCA1360, 1:2,000), $\alpha$-RIPK1 (BD Biosciences, 610459,1:1,000 for western blotting (WB) and 1:50 for immunofluorescence studies), $\alpha$-Actin (Santa Cruz, sc-1615, 1:4,000), $\alpha$-MYO7A (Developmental Studies Hybridoma Bank, 138-1-s, 1:1,000 for WB and 1:50 for immunofluorescence studies), $\alpha$-CASP8 for WB (MBL, M032-3, 1:5,000), $\alpha$-CASP8 to detect cleaved CASP8 (for human: R\&D, AF1650, 1:2,000; for mouse: Cell Signaling Technology, 9429, 1:1,000), rabbit and goat $\alpha$-CASP8 (Santa Cruz Biotechnology, sc-7890 and sc-6136, both 1:50 for immunofluorescence studies), CF488A-donkey $\alpha$-mouse IgG (Biotium, A21202, 1:1,000), CF633-donkey $\alpha$-rabbit IgG (Biotium, 20125, 1:1,000) and CF633-donkey $\alpha$-goat IgG (Biotium, 20127, 1:1,000). SM (SM164) was a gift from Shaomeng Wang (University of Michigan) and TNF was obtained from Enzo Life Sciences Uncropped WBs are shown in Supplementary Fig. 5.

Fly stocks. Flies were raised at $25^{\circ} \mathrm{C}$ according to standard procedures, unless stated otherwise. RNAi stocks were obtained from the Vienna Drosophila RNAi Center, with the exception of GFP RNAi (a gift from R. Ueda) and LacZ RNAi (a gift from M. Miura). UAS-GFP-ck TAIL was a gift from D. Kiehart. $U A S-D m I K K \varepsilon^{G 250 D}$ (DN-DmIKKe) and UAS-sgg46 ${ }^{D 235 G / D 300 G}$ were gifts from M. Miura. GMR-hid,ey-FLP were previously described ${ }^{18}$. All other stocks were obtained from the Bloomington Drosophila Stock Center or described previously.

Quantification of macrochaete. Crosses were performed at $18^{\circ} \mathrm{C}$. The macrochaete on the scutellum and the central portion of the notum were analysed. Any macrochaete that were present in addition to the usual numbers (four on the scutellum and four on the central part of the notum) were scored as ectopic macrochaete.

Border cell migration. Ovaries were dissected in PBS, fixed in $4 \%$ formaldehyde for $20 \mathrm{~min}$ and permeabilized in PBS containing $0.1 \%$ Tween 20 . Ovaries were stained with 4,6-diamidino-2-phenylindole and phalloidin (Invitrogen). After mounting in Vectashield Hardset Mounting Medium (Vector Laboratories), confocal images of stage-10 egg chambers were taken. The distance the border cells had moved was measured using ImageJ software in at least 30 stage-10 egg chambers and expressed as a percentage of the total distance from the anterior pole to the oocyte.

Tissue culture and confocal microscopy. Drosophila S2 cells and mammalian SKOV3, HT1080, NIH3T3 and Swiss3T3 were obtained from ATCC. SKOV3 $3^{\mathrm{CrmA}}$ were described in ref. 33. Small interfering RNA (siRNA) oligos are as follows: human Ripk1 siRNA: 5'-CCAACUAUCUAGGAAAUACtt- $3^{\prime}$; human Myo7A: $5^{\prime}$ GGACGGGUGUGUACUUUGU-3'; non-targeting: AllStars Negative control1027281 (Qiagen) (for mouse and human); mouse Myo7A-1: (Qiagen) Mm_Myo7a_1 FlexiTube siRNA, Cat:SI01322279; mouse Myo7A: (Qiagen) Mm_Myo7a_2 FlexiTube siRNA, Cat:SI01322286; mouse Ripk1: 4390771ID:s72977 (Ambion); and mouse Casp8 siGenome SMART pool (Thermo Scientific), Cat M-043044-01. siRNA oligos were transfected using HiPerFect (Qiagen) $48 \mathrm{~h}$ before experimental conditions. Drosophila S2 cells were transfected with Effectene (Qiagen) according to the manufacturer's instructions. For immunofluorescence staining of mammalian cells, $10^{5}$ cells were plated on $13 \mathrm{~mm}$ glass cover slips (VWR) and retro-transfected with $50 \mathrm{nmol}$ siRNA as indicated. Cells were then treated with the indicated biologics and fixed in $4 \%$ paraformaldehyde for $10 \mathrm{~min}$. Following $10 \mathrm{~min}$ permeabilization with PBS containing $0.5 \%$ Triton X-100, cells were incubated for $1 \mathrm{~h}$ in $5 \%$ BSA in PBS. Respective primary antibodies were then added as follows: $\alpha$-RIPK1 (BD Bioscience), rabbit or goat $\alpha$-CASP8 (Santa Cruz Biotechnology) and $\alpha$-MYO7A (Sigma). Alexa Fluor 633-conjugated phalloidin (Invitrogen) was used to stain actin filaments. Secondary fluorescent conjugated antibodies were then added as follows: $1: 1,000$ CF488A-donkey $\alpha$-mouse and 1:1,000 CF633-donkey $\alpha$-rabbit or 1:1,000 CF633-donkey $\alpha$-rabbit. Cells were visualized by confocal microscopy (objective $\times 40$, Zeiss LSM710). For Fig. 4c,d, SM was used at $100 \mathrm{nM}$ and SKOV3 cells were treated for 14-16 h. For Fig. 4f-i, TNF (Enzo Life Sciences) was used at $10 \mathrm{ng} \mathrm{ml}^{-1}$ (unless stated otherwise) in conjunction with $10 \mathrm{nM} \mathrm{SM}$ and cells were treated for $8 \mathrm{~h}$. Expression of CrmA was induced using $100 \mathrm{nM}$ Tamoxifen, at least $4 \mathrm{~h}$ before analysis.

Cell lysis and immunoprecipitation. Cells were lysed in $50 \mathrm{mM}$ Tris $\mathrm{pH} 7.5$, $150 \mathrm{mM} \mathrm{NaCl}, 0.1 \%$ Triton X-100, 10\% glycerol and $1 \mathrm{mM}$ EDTA. Where lysates were used for immunoprecipitations, complete protease inhibitor cocktail (Roche) was added. Immunoprecipitations were performed using $\alpha$-Myc or $\alpha$-HA-agarose beads (Sigma). Samples were examined by immunoblot analysis using either chemiluminescence (Amersham Biosciences) or Odyssey Infrared Technology (LI-COR Biosciences).

Proximity ligation assay. PLA was performed according to the manufacturer's protocol using the Duolink Detection Kit (Cambridge BioScience Ltd). Immunofluorescence staining of RIPK1 and CASP8 for the Duolink was carried out following the above-described protocol for immunofluorescence detection up until the primary antibody incubation step. Probe incubation, ligation and amplification reaction were carried out according to the manufacturer instructions. Cy3 signal amplification was used for the assay. Cells were examined with a confocal microscope (objective $\times 40$, Zeiss LSM 710).

FACS and DEVDase assays. For FACS and DEVDase assays, $10^{4}$ cells were plated in a 96-well plate and reverse siRNA transfection was performed with $50 \mathrm{nM}$ tota siRNA for $40 \mathrm{~h}$ ( $25 \mathrm{nM}$ of each individual siRNA balanced with control siRNA). Cells were treated with TNF $\left(500 \mathrm{pg} \mathrm{ml}^{-1}\right.$ or $\left.10 \mathrm{ng} \mathrm{ml}^{-1}\right)$ plus SM in $150 \mu \mathrm{l}$ for $24 \mathrm{~h}$ (FACS) or 5-6h (DEVDase). For FACS analysis, medium containing dead cells was transferred to a round-bottom 96-well plate, live cells were trypsinized in $50 \mu \mathrm{l}$, harvested with $100 \mu \mathrm{l}$ of medium containing $1 \mu \mathrm{g} \mathrm{ml}^{-1}$ propidum iodide (Sigma) and combined with dead cells (total volume $300 \mu \mathrm{l}$ ). The 96 -well plate was analysed by FACS using a plate reader. Data shown are from 5,000 cells per condition. For DEVDase assays, the medium was removed after treatment and $20 \mu 11 \%$ Triton lysis buffer was added to each well. Plates were placed at $-80^{\circ} \mathrm{C}$ to aid cell lysis. Plates were thawed at room temperature for $15 \mathrm{~min}$, after which $180 \mu \mathrm{l}$ DEVDase assay mix (20 $\mu \mathrm{M}$ Ac-DEVD-AMC (Sigma), $1 \mathrm{mM}$ dithiothreitol, $50 \mathrm{mM}$ Tris pH 7.5, $150 \mathrm{mM} \mathrm{NaCl}, 0.1 \%$ Triton X-100 and $5 \%$ glycerol) was added to each well (it is noteworthy that cell lysates were not cleared). Reactions were incubated at room temperature for up to $24 \mathrm{~h}$. DEVDase activity was read at $380 \mathrm{nM}$ excitation/460 nM emission.

Statistical analysis. For most experiments, data were analysed pairwise with two-tailed $t$-tests assuming equal variance, using the following website: http:// studentsttest.com. Macrochaete data were analysed with Fisher's exact test, using the following website: http://www.quantitativeskills.com/sisa/statistics/fiveby2.htm.

\section{References}

1. McIlwain, D. R., Berger, T. \& Mak, T. W. Caspase functions in cell death and disease. Cold Spring Harb. Perspect. Biol. 7 (2015).

2. Meier, P. \& Vousden, K. H. Lucifer's labyrinth--ten years of path finding in cell death. Mol. Cell 28, 746-754 (2007).

3. Miura, M. Apoptotic and non-apoptotic caspase functions in neural development. Neurochem. Res. 36, 1253-1260 (2011).

4. Fuchs, Y. \& Steller, H. Programmed cell death in animal development and disease. Cell 147, 742-758 (2011).

5. Kuranaga, E. \& Miura, M. Nonapoptotic functions of caspases: caspases as regulatory molecules for immunity and cell-fate determination. Trends Cell Biol. 17, 135-144 (2007)

6. Pop, C. \& Salvesen, G. S. Human caspases: activation, specificity, and regulation. J. Biol. Chem. 284, 21777-21781 (2009)

7. Mathur, P. \& Yang, J. Usher syndrome: hearing loss, retinal degeneration and associated abnormalities. Biochim. Biophys. Acta 1852, 406-420 (2015).

8. Williams, D. S. Usher syndrome: animal models, retinal function of Usher proteins, and prospects for gene therapy. Vision Res. 48, 433-441 (2008).

9. Kiehart, D. P. et al. Drosophila crinkled, mutations of which disrupt morphogenesis and cause lethality, encodes fly myosin VIIA. Genetics 168, 1337-1352 (2004).

10. Quinn, L. M. et al. An essential role for the caspase dronc in developmentally programmed cell death in Drosophila. J. Biol. Chem. 275, 40416-40424 (2000).

11. Meier, P., Silke, J., Leevers, S. J. \& Evan, G. I. The Drosophila caspase DRONC is regulated by DIAP1. EMBO J. 19, 598-611 (2000).

12. Hawkins, C. J., Yoo, S. J., Peterson, E. P., Wang, S. L., Vernooy, S. Y. \& Hay, B. A. The Drosophila caspase DRONC cleaves following glutamate or aspartate and is regulated by DIAP1, HID, and GRIM. J. Biol. Chem. 275, 27084-27093 (2000).

13. Goyal, L., McCall, K., Agapite, J., Hartwieg, E. \& Steller, H. Induction of apoptosis by Drosophila reaper, hid and grim through inhibition of IAP function. EMBO J. 19, 589-597 (2000).

14. Wang, S. L., Hawkins, C. J., Yoo, S. J., Muller, H. A. \& Hay, B. A. The Drosophila caspase inhibitor DIAP1 is essential for cell survival and is negatively regulated by HID. Cell 98, 453-463 (1999).

15. Wing, J. P., Zhou, L., Schwartz, L. M. \& Nambu, J. R. Distinct cell killing properties of the Drosophila reaper, head involution defective, and grim genes. Cell Death Differ. 5, 930-939 (1998).

16. Bergmann, A., Agapite, J., McCall, K. \& Steller, H. The Drosophila gene hid is a direct molecular target of Ras-dependent survival signaling. Cell 95, 331-341 (1998)

17. Grether, M. E., Abrams, J. M., Agapite, J., White, K. \& Steller, H. The head involution defective gene of Drosophila melanogaster functions in programmed cell death. Genes Dev. 9, 1694-1708 (1995).

18. Xu, D., Li, Y., Arcaro, M., Lackey, M. \& Bergmann, A. The CARD-carrying caspase Dronc is essential for most, but not all, developmental cell death in Drosophila. Development 132, 2125-2134 (2005). 
19. Baum, J. S., Arama, E., Steller, H. \& McCall, K. The Drosophila caspases Strica and Dronc function redundantly in programmed cell death during oogenesis. Cell Death Differ. 14, 1508-1517 (2007).

20. Kuranaga, E. et al. Drosophila IKK-related kinase regulates nonapoptotic function of caspases via degradation of IAPs. Cell 126, 583-596 (2006).

21. Oshima, K. et al. IKK epsilon regulates F actin assembly and interacts with Drosophila IAP1 in cellular morphogenesis. Curr. Biol. 16, 1531-1537 (2006).

22. Cullen, K. \& McCall, K. Role of programmed cell death in patterning the Drosophila antennal arista. Dev. Biol. 275, 82-92 (2004).

23. Wolff, T. \& Ready, D. F. The beginning of pattern formation in the Drosophila compound eye: the morphogenetic furrow and the second mitotic wave. Development 113, 841-850 (1991).

24. Mendes, C. S. et al. Cytochrome c-d regulates developmental apoptosis in the Drosophila retina. EMBO Rep. 7, 933-939 (2006).

25. Bergmann, A., Agapite, J. \& Steller, H. Mechanisms and control of programmed cell death in invertebrates. Oncogene 17, 3215-3223 (1998).

26. Abrams, J. M., White, K., Fessler, L. I. \& Steller, H. Programmed cell death during Drosophila embryogenesis. Development 117, 29-43 (1993).

27. Kanuka, H. et al. Drosophila caspase transduces Shaggy/GSK-3beta kinase activity in neural precursor development. EMBO J. 24, 3793-3806 (2005).

28. Todi, S. V., Franke, J. D., Kiehart, D. P. \& Eberl, D. F. Myosin VIIA defects, which underlie the Usher 1B syndrome in humans, lead to deafness in Drosophila. Curr. Biol. 15, 862-868 (2005).

29. Geisbrecht, E. R. \& Montell, D. J. A role for Drosophila IAP1-mediated caspase inhibition in Rac-dependent cell migration. Cell 118, 111-125 (2004).

30. Ragab, A., Buechling, T., Gesellchen, V., Spirohn, K., Boettcher, A. L. \& Boutros, M. Drosophila Ras/MAPK signalling regulates innate immune responses in immune and intestinal stem cells. EMBO J. 30, 1123-1136 (2011).

31. Blanco-Sanchez, B., Clement, A., Fierro, Jr J., Washbourne, P. \& Westerfield, M. Complexes of Usher proteins preassemble at the endoplasmic reticulum and are required for trafficking and ER homeostasis. Dis. Model Mech. 7, 547-559 (2014).

32. Soderberg, O. et al. Direct observation of individual endogenous protein complexes in situ by proximity ligation. Nat. Methods 3, 995-1000 (2006)

33. Tenev, T. et al. The Ripoptosome, a signaling platform that assembles in response to genotoxic stress and loss of IAPs. Mol. Cell 43, 432-448 (2011).

34. Feoktistova, M. et al. cIAPs block Ripoptosome formation, a RIP1/caspase- 8 containing intracellular cell death complex differentially regulated by cFLIP isoforms. Mol. Cell 43, 449-463 (2011).

35. Wang, L., Du, F. \& Wang, X. TNF-alpha induces two distinct caspase- 8 activation pathways. Cell 133, 693-703 (2008).

36. Chan, F. K. et al. A role for tumor necrosis factor receptor-2 and receptorinteracting protein in programmed necrosis and antiviral responses. J. Biol. Chem. 278, 51613-51621 (2003).

37. Lin, Y., Devin, A., Rodriguez, Y. \& Liu, Z. G. Cleavage of the death domain kinase RIP by caspase- 8 prompts TNF-induced apoptosis. Genes Dev. 13, 2514-2526 (1999).

38. Oberst, A. et al. Catalytic activity of the caspase-8-FLIP(L) complex inhibits RIPK3-dependent necrosis. Nature 471, 363-367 (2011).

39. Green, D. R., Oberst, A., Dillon, C. P., Weinlich, R. \& Salvesen, G. S. RIPK-dependent necrosis and its regulation by caspases: a mystery in five acts. Mol. Cell 44, 9-16 (2011).

40. Silke, J. \& Meier, P. Inhibitor of apoptosis (IAP) proteins-modulators of cell death and inflammation. Cold Spring Harb. Perspect. Biol. 5 (2013).

41. Walczak, H. TNF and ubiquitin at the crossroads of gene activation, cell death, inflammation, and cancer. Immunol. Rev. 244, 9-28 (2011).

42. Micheau, O. \& Tschopp, J. Induction of TNF receptor I-mediated apoptosis via two sequential signaling complexes. Cell 114, 181-190 (2003).

43. Balkwill, F. Tumour necrosis factor and cancer. Nat. Rev. Cancer 9, 361-371 (2009).
44. Pasparakis, M. \& Vandenabeele, P. Necroptosis and its role in inflammation. Nature 517, 311-320 (2015).

45. Al-Gayyar, M. M. \& Elsherbiny, N. M. Contribution of TNF-alpha to the development of retinal neurodegenerative disorders. Eur. Cytokine Netw. 24, 27-36 (2013).

46. Williams, D. S. \& Lopes, V. S. The many different cellular functions of MYO7A in the retina. Biochem. Soc. Trans. 39, 1207-1210 (2011).

47. Dickens, L. S. et al. A death effector domain chain DISC model reveals a crucial role for caspase- 8 chain assembly in mediating apoptotic cell death. Mol. Cell 47, 291-305 (2012).

\section{Acknowledgements}

We are indebted to Ryu Ueda, Masayuki Miura and Daniel Kiehart for reagents and fly strains, and Shaomeng Wang for the gift of SMAC mimetic compounds. We thank members of the Cell Death and Inflammation Team for support and critical reading of the manuscript. We are very grateful to Frederik Wallberg, Brian Stramer, John Davis, Eunice Chan, Alice Genevet, Nic Tapon and Yossi Kaplan for technical assistance and helpful discussions. P.M. is supported by Breakthrough/Breast Cancer NOW, AICR, MRC and Komen promise grants. A.B. is supported by a grant from the National Institute of General Medical Science (NIGMS). D.W. is supported by the National Institutes of Health (grant number EY07042). N.M. is funded by Breast Cancer Now (2013MayPR023). We acknowledge NHS funding to the NIHR Biomedical Research Centre.

\section{Author contributions}

M.H.O. designed and performed experiments shown in Figs 2a,b, 3b-e, 5c-e,h and Supplementary Figs 1e,h and 2a-e. S.W.-J. designed and performed experiments shown in Figs 1b-f, 4a,b, 6a,b and Supplementary Fig. 3a,b. G.L. designed and conducted the experiments shown in Figs $6 \mathrm{c}, \mathrm{d}, 7 \mathrm{a}-\mathrm{c}$ and Supplementary Fig. $4 \mathrm{a}-\mathrm{f}$. N.M. and P.S.R. conducted and analysed the experiments shown in Fig. 5f,g. R.F. designed and conducted the experiments shown in Fig. 8a-c. L.A. and E.A. conducted and analysed the experiments shown in Supplementary Fig. 3e. O.M. and C.M.D. designed, conducted and analysed the experiments shown in Supplementary Figs 3c,d and 1i, respectively. M.T. and A.B. designed, conducted and analysed the data shown in Fig. 2a and Supplementary Fig. 1a-d. A.W. and M.G. conducted the mass spectrometry experiment and analysed the data. D.W. provided the complementary DNA of MYO7A. D.R. conducted the scanning electron microscopy picture shown in Supplementary Fig. 1i. M.H.O., S.W.-J., G.L., R.F., E.A., O.M., C.M.D., A.B., M.G., D.W., P.S.R., T.T., R.W., K.B. and P.M. analysed data, provided helpful suggestions and discussions. M.H.O. and P.M. designed the study and wrote the manuscript.

\section{Additional information}

Supplementary Information accompanies this paper at http://www.nature.com/ naturecommunications

Competing financial interests: The authors declare no competing financial interests.

Reprints and permission information is available online at http://npg.nature.com/ reprintsandpermissions/

How to cite this article: Orme, M. H. et al. The unconventional myosin CRINKLED and its mammalian orthologue MYO7A regulate caspases in their signalling roles. Nat. Commun. 7:10972 doi: 10.1038/ncomms10972 (2016).

This work is licensed under a Creative Commons Attribution 4.0 International License. The images or other third party material in this article are included in the article's Creative Commons license, unless indicated otherwise in the credit line; if the material is not included under the Creative Commons license, users will need to obtain permission from the license holder to reproduce the material. To view a copy of this license, visit http://creativecommons.org/licenses/by/4.0/ 